\title{
Ocorrência de espécies arbóreas em áreas urbanas e suburbanas de Três Lagoas, MS
}

\author{
Occurrence of trees in central and peripherical areas in Três Lagoas, MS \\ Alessandra Aparecida Santos' \\ ' Graduada em biologia, Universidade federal de Mato Grosso do Sul
}

\section{Resumo}

Áreas urbanas são constituídas por um mosaico de espaços físicos, ecológicos e sociais. Com o aumento da urbanização a alteração das áreas verdes nas cidades tende a aumentar. O objetivo deste estudo foi investigar as variações na ocorrência e distribuição de espécies arbóreas em áreas suburbanas e urbanas da cidade de Três Lagoas, MS (Brasil). Este estudo foi desenvolvido de Julho/2012 a Novembro/2012 quando foram selecionados aleatoriamente quatro transectos paralelos distanciados por no mínimo 500 metros. A amostragem foi realizada sistematicamente em quarteirões intercalados a cada 200 metros onde foram registrados: a) o número de indivíduos arbóreos, b) as espécies, c) origem, d) DAP, e) altura, f) data e local. Foram encontrados 336 indivíduos pertencentes a 44 espécies arbóreas de 21 famílias botânicas nas áreas urbanas e suburbanas. Também, 14 (32 \%) das espécies registradas eram exóticas e 30 (68\%) nativas. A ampla variação da riqueza e densidade de espécies demonstra que a ocorrência das árvores nas calçadas depende altamente das decisões dos residentes. Futuros estudos sobre a dinâmica da comunidade vegetal em áreas urbanas devem considerar a origem das espécies segundo não apenas ao bioma, mas também segundo as fitofisionomias nas quais ocorrem.

Palavras-chaves: Arborização, Planejamento urbano, Áreas urbanas e suburbanas.

\begin{abstract}
Urban areas are constituted by a patch of physical, ecological and social spaces. By the increasing urbanization, the conversion of green areas in cities tends to grow as well. The goal of this work was investigate variations in the occurrence and distribution of tree species in suburban and urban areas in Três Lagoas municipality, MS (Brasil). This study was conducted from July to November/2012, when four parallel transects were randomly selected and spaced at least by $500 \mathrm{~m}$ away from each other. The sampling was carried out systematically in interspersed blocks (200 m), where it was recorded: a) the number of trees, b) its species, c) its origin, d) its DBH, e) its height, f) time and place. I found 336 individuals, from which 44 tree species belonged to 21 families in urban and suburban areas. Also, 14 ( $32 \%$ ) of the species recorded were exotic and $30(68 \%)$ were native ones. The wide variation of species density and richness shows that trees occurrence on sidewalks depends highly on the residents decisions. Future studies concerning the dynamic of vegetal community in urban areas should consider the local origin of the species according to its vegetal physiognomies rather than only its biome where they occur.
\end{abstract}

Keywords: Arborization, Urban planning; Urban and suburban areas. 


\section{INTRODUÇÃO}

Áreas urbanas são constituídas por um mosaico de espaços físico, ecológico e social (ZIPPERER et al., 1997). Com o aumento global da urbanização a conversão da cobertura vegetal para uso humano tende a aumentar, alterando os padrões e processos ecológicos das comunidades vegetais dos ecossistemas (GRIMM et al., 2000). Ecossistemas urbanos são altamente dinâmicos e estão em constante mudança (GILBERT, 1989; ADAMS, 1994; SAVARD et al., 2000). Também, são áreas caracterizadas por elevado nível de heterogeneidade estrutural, frequentemente, organizadas ao longo de gradientes que se estendem desde as paisagens adjacentes até o centro urbano (MCDONNELL \& PICKETT, 1990; MCDONNELL et a1., 1993). Entretanto, o processo de urbanização frequentemente pode ser um dos maiores fatores de homogeneização de áreas naturais (MCKINNEY, 2006), em que a riqueza de espécies tende a diminuir com o avanço da urbanização (BLAIR, 1996). Uma vez que o processo de urbanização é crescente e irreversível, é preciso conciliar o desenvolvimento dessas áreas com a conservação ambiental, proporcionando assim a melhoria na qualidade de vida das populações urbanas (ALBERTIN et al., 2011).

Enquanto a diversidade e a abundância de espécies são frequentemente relacionadas à qualidade da vida urbana (ADAMS, 1994), a superabundância de algumas espécies pode ser indesejável (COOPER, 1987). A invasão de espécies exóticas e a superabundância pode diminuir a diversidade local pela substituição de espécies nativas por espécies de interesse da espécie humana (DONNELLY \& MARZLUFF, 2004). Por outro lado, um dos primeiros passos do processo de urbanização tende a ser a remoção da vegetação, levando consequentemente à erradicação das árvores típicas da região, acarretando o desequilíbrio ambiental, criando marcantes obstáculos para o desenvolvimento de novas espécies vegetais e comprometendo os padrões de qualidade ambiental bem como o de uma vida saudável à população humana (SILVA, 2000). Consequentemente, o planejamento da composição e estrutura arbórea em áreas urbanas deve ser apreciado com atenção segundo cada cidade e sua vegetação típica circundante.

Entre os objetivos do planejamento de áreas urbanas está o desenvolvimento de locais mais amenos e adequados à sobrevivência humana (PICKETT et al., 2001). Porém, o planejamento urbano certamente requer informações mais abrangentes sobre os fatores que afetam a distribuição das espécies e a estrutura de comunidades arbóreas urbanas no sentido de criar e manter a biodiversidade nessas áreas. Nesse sentido, a arborização deve ser objeto de planejamento prévio de modo que a torne compatível com o uso que o homem faz da área urbana.

Os esforços de conservação e restauração relacionados às espécies presentes na área urbana atualmente se concentram em desenvolver a participação de cidadãos na conservação das espécies urbanas, aprimorar a qualidade de vida dos habitantes urbanos e educá-los sobre os conceitos ecológicos relacionados às espécies das quais fazem uso (GILBERT, 1989; ADAMS, 1994; NIEMELÄ, 1999). Consequentemente, a análise da dinâmica da vegetação dentro da paisagem urbana deve considerar as influências das decisões humanas (ZIPPERER et al., 1997).

De fato, investigações sobre a vegetação urbana em paisagens urbanizadas e as decisões tomadas por grande número de pessoas têm sido conduzidas em escalas locais (PROFOUS \& LOEB, 1984; RUDNICKY \& MCDONNELL, 1989) e regionais (NOWAK, 1994). Portanto, as decisões dos residentes de uma cidade podem ter um maior impacto na composição vegetal da cidade em uma escala local, enquanto os planos diretores sobre o planejamento da arborização da cidade tendem a promover um maior impacto em escala regional (HOSTETLER, 1999). GUIMARÃES (2006) afirma que é preciso encontrar estratégias, dentro dos centros urbanos, que permitam compatibilizar as exigências da vida humana e as necessidades ecológicas de outras espécies de forma a possibilitar a sobrevivência de uma maior diversidade de organismos.

Consequentemente, caracterizar a comunidade arbórea urbana bem como identificar os fatores que influenciam as variações na distribuição vegetal nas cidades pode favorecer o planejamento da arborização das áreas urbanas. Portanto, neste estudo foram investigadas as variações da ocorrência e distribuição arbórea em áreas urbanas e suburbanas da cidade de Três Lagoas, MS (Brasil). 


\section{MATERIAIS E MÉTODOS}

Este estudo foi desenvolvido de julho/2012 a novembro/2012 em áreas urbanas de Três Lagoas (Mato Grosso do Sul, Brasil; 51 ${ }^{\circ} 48^{\prime} \mathrm{W}, 20^{\circ} 46^{\prime} \mathrm{S}, 340 \mathrm{~m}$ ). Para tanto, a cidade foi dividida em oito grandes áreas nomeadas pelo bairro principal, com o auxílio de um mapa das quais quatro (Centro/ Santos Dumont, Paranapungá/ Vila Nova, Alvorada/Colinos e Lapa/Interlagos) foram sorteadas para servir como local de amostragem. O clima é do tipo sazonal com média anual de precipitação de $1400 \mathrm{~mm}$ (70\% ocorrendo durante a estação chuvosa). A média anual de temperatura está em torno de $24^{\circ} \mathrm{C}$ na estação chuvosa e $17^{\circ} \mathrm{C}$ na seca. A vegetação ao redor da área urbana inclui um mosaico de pastagens com remanescentes de cerrado e floresta estacional. A vegetação urbana em si é variável, característica típica de áreas urbanas devido ao uso das áreas e contexto social (PICKETT et al., 2001).

Para avaliar a composição arbórea de Três Lagoas foram selecionados aleatoriamente quatro transectos estabelecidos paralelamente nas ruas ou avenidas, distanciados por no mínimo 500 metros para assegurar a independência das composições arbóreas segundo as decisões dos residentes. Uma vez que os residentes influenciam em grande parte a composição arbórea das cidades, essa distância mínima adotada diminuiria ao máximo a influência das escolhas arbóreas determinadas por residentes donos de múltiplas propriedades ou ainda o efeito de quarteirões dominados por grandes empresas (que frequentemente tomam um quarteirão inteiro ou dois). Nesse sentido, essa distância entre os transectos foi feita de forma a favorecer a amostragem das quatro grandes bairros/locais da cidade que contemplam áreas urbanas e suburbanas. A amostragem foi feita sistematicamente em quarteirões intercalados a cada 200 metros (quadras) de forma a assegurar a independência da amostragem das árvores localizadas em cada área. Durante quatro meses cada um dos quatro transectos foi percorrido para a amostragem da ocorrência de árvores (tanto nas calçadas quanto nos canteiros urbanos das avenidas) no período entre 7: $00 \mathrm{~h}$ e 17:00 h. Os transectos foram categorizados em ruas principais ou avenidas e ruas periferais para representar áreas urbanas e suburbanas da cidade, respectivamente. Nesse caso, a avaliação da composição arbórea das ruas foi considerada a partir da amostragem das calçadas e os canteiros centrais (nos casos das avenidas). Para analisar a relação entre a abundância e local (áreas urbanas ou suburbanas) em cada quarteirão foi amostrada a densidade e a riqueza de espécies arbóreas. Também, o diâmetro à altura do peito foi medido (DAP). As espécies arbóreas foram identificadas através de comparação com exemplares presentes no herbário da UFMS. A nomenclatura seguiu Lorenzi (1998). A análise das variações do DAP, densidade arbórea bem como das proporções de espécies nativas e exóticas em áreas urbanas e suburbanas foi usado o teste não-paramétrico de Mann-Whitney devido à ausência de normalidade dos dados.

\section{RESULTADOS E DISCUSSÃO}

Foram encontrados 336 indivíduos pertencentes a 44 espécies arbóreas de 21 famílias nas áreas urbanas e suburbanas de Três Lagoas (Tabela 1) das quais 14 (32\%) eram exóticas e 30 (68\%) nativas. Entre as espécies mais comuns estão Licania tomentosa (Benth.) Fritsch, Syagrus oleracea (Mart.) Becc., Pinus echinatta (Mill.), Tabebuia roseoalba (Ridl.) Sandwith, Ficus microcarpa L.f., Cassia leiandra Benth. e Hibiscus tilliaceus L. A riqueza de espécies por quadra variou de 1 a 15 espécies com média de $6.70 \pm 4.6$ e a variação do número de espécies entre áreas urbanas e suburbanas foi semelhante $(\mathrm{U}=20.5, \mathrm{p}=0.14)$. A densidade de indivíduos encontrados variou de 1 a 197 árvores por quadra com média de árvores de $16.8 \pm 13.9$, cuja variação em áreas urbanas e suburbanas foi significativamente semelhante $(\mathrm{U}=27, \mathrm{p}=0.23)$.

A proporção de árvores exóticas e nativas em áreas urbanas e suburbanas da cidade foi significativamente semelhante $(\mathrm{U}=37.5, \mathrm{p}=0.33, \mathrm{U}=26, \mathrm{p}=0.07$; respectivamente, Figura 1$)$.

O DAB médio foi de $19.55 \pm 13.71 \mathrm{~cm}$ e não variou entre áreas urbanas e suburbanas $(U=6, \mathrm{p}=$ 0.11). O DAP médio representa indivíduos reprodutivamente maduros, enquanto a altura demonstra a presença de árvores naturalmente, ou na maioria das vezes, artificialmente baixas devido à intervenção humana das podas.

A ampla variação da riqueza e densidade de espécies por quadra (de 1 a 15, e de 1 a 197; respectivamente) demonstra que a ocorrência dos indivíduos arbóreos nas calçadas depende altamente 
das decisões dos residentes que escolhem as espécies e o número de indivíduos arbóreos que plantam em frente às suas residências ou mesmo nos canteiros centrais. Porém, mais do que apenas considerar a densidade absoluta de espécies nas áreas urbanas ou ainda a riqueza para efeito de planejamento da arborização urbana, é importante considerar a origem das espécies arbóreas tendo em vista a tendência das cidades abrigarem um alto número de espécies exóticas proporcionalmente às nativas (PICKETT et al. 2001). Por outro lado, segundo MENEGAT (1999) 47\% das espécies plantadas no país são nativas,

Tabela 1. Espécie, número de indivíduos e origem (Nat= nativas e Ex= exóticas) das árvores registradas em áreas urbanas e suburbanas de Três Lagoas em 2012

\begin{tabular}{|c|c|c|c|c|c|}
\hline Espécies arbóreas & Origem & $\begin{array}{l}\mathrm{N}^{\circ} \mathrm{de} \\
\text { indivíduos (\%) }\end{array}$ & Espécies arbóreas & Origem & $\begin{array}{l}\mathrm{N}^{\circ} \text { de } \\
\text { indivíduos (\%) }\end{array}$ \\
\hline Anonaceae & & & Fabaceae & & \\
\hline Annona muricata L. & Ex & $3(0.9)$ & Vatairea macrocarpa (Benth.) Ducke & Nat & $2(0.6)$ \\
\hline Annona squamosa $\mathrm{L}$. & Ex & $2(0.6)$ & Bauhinia longifolia (Bong.) Steud & Nat & $4(1.2)$ \\
\hline Apocynaceae & & & Peltophorum dubium (Spreng.) Taub & Nat & $2(0.6)$ \\
\hline Hancornia speciosa Gomes & Nat & $3(0.9)$ & Delonix regia Raff & Ex & $2(0.6)$ \\
\hline Tabernaemontana divaricata $L$. & Ex & $2(0.6)$ & Clitoria fairchildiana R.A. Howard & Nat & $2(0.6)$ \\
\hline Areaceae & & & Anadenanthera falcata (Benth.) Speg & Nat & $1(0.3)$ \\
\hline $\begin{array}{l}\text { Acrocomia aculeta (Jacq.) Lodd. ex } \\
\text { Mart. }\end{array}$ & Nat & $3(0.9)$ & Dimorphandra mollis Benth. & Nat & $1(0.3)$ \\
\hline Syagrus oleracea (Mart.) Becc. & Nat & $17(5)$ & Bignoniaceae & & \\
\hline Araliaceae & & & Handroanthus impetiginosa (Vell.) Mattos & Nat & $2(0.6)$ \\
\hline $\begin{array}{l}\text { Schefflera macrocarpa (Cham. \& } \\
\text { Schltdl.) }\end{array}$ & Nat & $1(0.3)$ & Tabebuia roseoalba (Ridl.) Sandwith & Nat & $2(0.6)$ \\
\hline Anacardiaceae & & & Handroanthus ochraceus (Cham.) Mattos & Nat & $14(4.1)$ \\
\hline Annacardium occidentale $\mathrm{L}$. & Nat & $2(0.6)$ & Jacaranda cuspidifolia Mart. & Nat & $3(0.9)$ \\
\hline Mangifera indica $\mathrm{L}$. & Ex & $2(0.6)$ & Malvaceae & & \\
\hline Spondias purpúrea $\mathrm{L}$. & Nat & $6(1.8)$ & Ceiba speciosa (A. St.- Hil) Ravena & Nat & $1(0.3)$ \\
\hline Schinus molle L. & Nat & $2(0.6)$ & Hibiscus tiliaceus L. & Ex & $5(1.5)$ \\
\hline Caesalpinoidae & & & Pachira aquática Aubl. & Nat & $3(0.9)$ \\
\hline Albizia lebbeck (L.) & Ex & $3(0.9)$ & Malpighiaceae & & \\
\hline Caesalpinia echinata (Lam.) Spreng. & Nat & $1(0.3)$ & Malpighia emarginata DC. & Nat & $1(0.3)$ \\
\hline Cassia leiandra Benth. & Nat & $5(1.5)$ & Moraceae & & \\
\hline Cassia grandis L. f. & Nat & $3(0.9)$ & Ficus microcarpa Vahl. & Ex & $6(1.8)$ \\
\hline Caricaceae & & & Morus nigra L. & Ex & $1(0.3)$ \\
\hline Carica papaya L. & Ex & $1(0.3)$ & Byrsonima coccolobifolia Kunth & Nat & $1(0.3)$ \\
\hline Inga laurina (Sw.) Willd. & Nat & $4(1.2)$ & Lythraceae & & \\
\hline Chrysobalanaceae & & & Punica granatum $\mathrm{L}$. & Ex & $1(0.3)$ \\
\hline Licania tomentosa (Benth.) Fritsch. & Nat & $197(58.6)$ & Myrtacea & & \\
\hline Caryocaricae & & & Psidium guajava L. & Nat & $4(1.2)$ \\
\hline Caryocar brasiliensis Cambes. & Nat & $2(0.6)$ & Rutaceae & & \\
\hline Combretacea & & & Citrus limonia (L.) Osbeck & Ex & $3(0.9)$ \\
\hline Terminalia catappa $\mathrm{L}$. & Ex & $1(0.3)$ & Pinaceae & & \\
\hline Vochysiaceae & & & Pinnus echinata Mill. & Ex & $12(3.6)$ \\
\hline Qualea parviflora Mart. & Nat & $1(0.3)$ & & & \\
\hline \multicolumn{6}{|l|}{ Sapotaceae } \\
\hline Pouteria torta (Mart.) Radlk. & Nat & $2(0.6)$ & & & \\
\hline Total & & & & & $336(100 \%)$ \\
\hline
\end{tabular}

Fonte: próprio autor. 


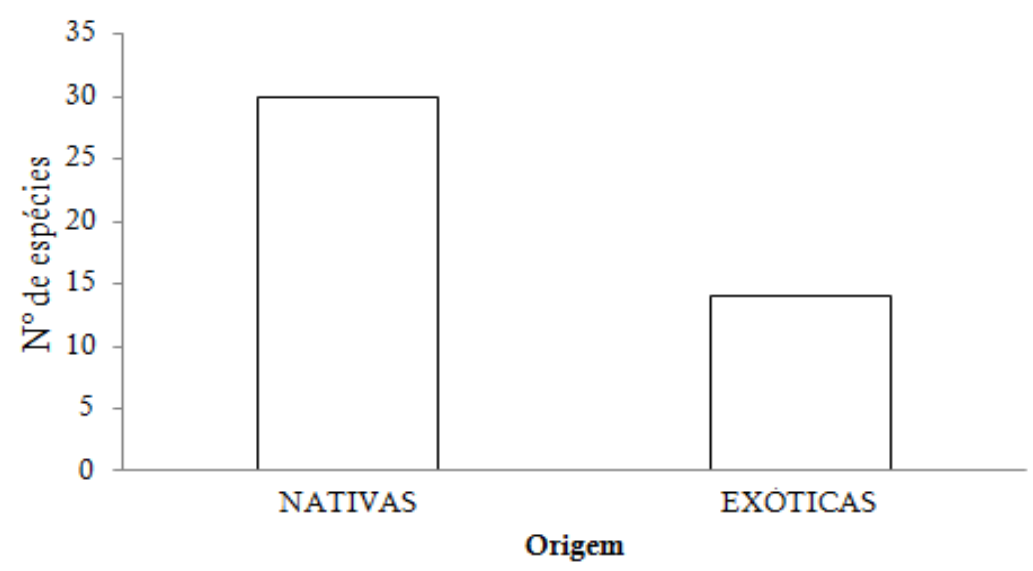

Figura 01. Número de espécies segundo a sua origem.

Fonte. Próprio autor

porém em Três Lagoas esse valor relativo apenas às espécies arbóreas sobe para $68 \%$, demonstrando que a cidade parece considerar a origem das espécies arbóreas em seus planos de arborização seja por parte dos residentes ou dos órgãos responsáveis pela conservação e manutenção das áreas públicas. Assim, espécies como Licania tomentosa (Benth.) Fritsch são comuns em várias cidades do Brasil.

Entretanto, essa árvore é típica de regiões de Mata Atlântica e apresenta uma congênere típica de Cerrado (Licania humilis Cham.), cujas características se assemelham muito à primeira que é amplamente utilizada para arborização de diversas cidades, independentemente do bioma no qual se localizam. Assim, não devemos considerar apenas a origem das espécies, mas também, as condições da fitofisionomia na qual se encontram. Por exemplo, espécies de cerrado senso restrito apresentam tipicamente porte mais baixo do que as de cerradão, o que favoreceria a arborização das cidades devido à constante preocupação com a rede elétrica. Isso porque árvores tipicamente mais baixas exigiriam um trabalho de poda menos oneroso e de menos impacto sobre a arquitetura natural da copa da espécie, e ainda, evitaria conflitos gerados pela interferência nas linhas de transmissão de energia. Surpreendentemente, o número de espécies nativas encontradas em Três Lagoas foi claramente maior do que de exóticas, sinalizando que as espécies típicas do Cerrado são altamente valorizadas pelos moradores da região, muito possivelmente pelo uso de seus frutos e sementes para posterior consumo, ou formato da copa usado para ornamentação das residências. Árvores pioneiras tipicamente tendem a dominar mais rapidamente uma determinada área, como Cecropia pachystachya Trécul., Fícus ssp. entre outras (LORENZI, 2000). Como consequência, é importante considerar essa informação ao elaborar planos de manejo para a arborização das áreas urbanas, como canteiros em áreas urbanas das cidades, por parte das instituições públicas relacionadas ao planejamento e manejo ambiental de áreas urbanas. Por outro lado, como aparentemente a maioria das árvores nas calçadas em áreas urbanas ou suburbanas é mantida pelos residentes, é de extrema importância enfocar ações de educação ambiental voltadas à conscientização dos moradores sobre a necessidade de se priorizar espécies nativas.

As áreas urbanas são locais de importante função ecológica por servirem de corredores para diversas espécies animais como aves, que se utilizam de espécies arbóreas como abrigo ou fonte de alimento (SAVARD et al., 2000). Consequentemente, o uso intensivo de espécies vegetais nativas associadas a espécies exóticas para arborização pode favorecer a atividade alimentar de animais que usam as áreas urbanas em sua rota de alimentação, como aves (SANTOS \& RAGUSA-NETTO in prep.). Entretanto, a estrutura vegetal pode determinar as condições para a sobrevivência das mais diversas espécies que se utilizem dessas áreas (PICKETT et al., 2001). Nesse sentido, um padrão emerge em que as variações de DAP, proporção de espécies exóticas e nativas, bem como riqueza e densidade de espécies arbóreas se apresentaram similares em áreas tanto urbanas quanto suburbanas. Tal fato demonstra o quanto Três Lagoas parece ser constituída por áreas estruturalmente homogêneas em relação ás comunidades arbóreas, apesar de apresentar microhábitats constituídos por espécies herbáceas que merecem maior atenção em futuros estudos sobre a dinâmica das comunidades animais que interagem com essas plantas dentro da cidade. 


\section{CONCLUSÕES}

A cidade de Três Lagoas apresenta uma arborização predominantemente nativa demonstrando o uso intenso dessas espécies para arborização tanto residencial quanto pública. A semelhança da riqueza de espécies, densidade e proporção de espécies nativas e exóticas entre áreas urbanas e suburbanas sugere que o padrão de arborização além de homogêneo, pode resultar, ao menos em parte, das decisões dos residentes e do manejo dos órgãos responsáveis visando a ornamentação e melhora da sensação térmica pelo sombreamento que as árvores garantem aos transeuntes. Futuros estudos sobre a dinâmica da comunidade vegetal em áreas urbanas devem considerar não somente a densidade relativa de indivíduos segundo a espécie, mas também sua origem. Essa última, deve ser estudada e discutida nos planos de manejo das cidades considerando as espécies segundo o bioma no qual a cidade se encontra o sentido de favorecer o uso de espécies nativas nas cidades.

\section{REFERÊNCIAS}

ALBERTIN, R. M.; ANGELIS, R.; NETO, G. A.; ANGELIS, B. L. D. Diagnóstico quali-quantitativo da arborização viária de nova esperança, Paraná, Brasil. Sociedade Brasileira de Arborização Urbana. v.6, n.3, p.128-148, 2011.

ADAMS, L. W. Urban Wildlife Habitats, a Landscape Perspective. University of Minnesota Press, Minneapolis, p. 208, 1994.

BLAIR, R. B. Land Use and Avian Species Diversity Along an Urban Gradient.

Ecological Applications, v. 6, n. 2., p. 506-519, 1996.

COOPER, J. A. Canada Goose Management at the Minneapolis Đ St. Paul International Airport. In: Adams, L. W.; Leedy, D. L. (eds.). Wildlife Conservation in Metropolitan Environments. National Institute for Urban Wildlife, Columbia, Maryland, p. 175-184, 1987.

DONNELLY, R., MARZLUFF, J. M. Importance of reserve size and landscape context to urban bird conservation. Conservation Biology, v. 18, p. 733-745, 2004.

GILBERT, O.L. The Ecology of Urban Habitats. Chapman \& Hall, Cambridge, UK, p. 369, 1989.

GRIMM, N. B., GROVE, J. M.; PICKETT, S. T. A.; REDMAN, C. L. Integrated approaches to long-term studies of urban ecological systems. BioScience, v. 50, p. 571-584, 2000.

GUIMARÃES, M. Há mais aves nos grandes centros urbanos hoje? Ciência \& Cultura. v. 58, n. 2, 2006.

HOSTETLER, M. Scale, birds, and human decisions: a potential for integrative research in urban ecosystems. Landscape and Urban Planning, v. 45, p. 15-19, 1999.

LORENZI, H. Árvores Brasileiras: Manual de identificação e cultivo de plantas arbóreas nativas do Brasil. v. 1, Editora Plantarum, Nova Odessa, p. 384, 2000.

MCDONNELL, M. J.; PICKETT, S. T. A. Ecosystem structure and function along urban rural gradients: an unexplored opportunity for ecology. Ecology, v. 71, p. 1232-1237, 1990.

MCDONNELL, M. J.; PICKET, S. P. A.; POUYAT, R. V. The application of the ecological gradient paradigm to the study of urban effects. In: McDonnell, M. J.; Pickett, S. T. A. (eds.). Human as Components of Ecosystems. Springer, New York, p. 175-189, 1993.

MCKINNEY, M. L. Urbanization as a major cause of biotic homogenization. Biological conservation. v.127, 
p. 247-260, 2006.

MENEGAT, R.; PORTO, M. L.; CARRARO, C. C.; FERNANDES, L. A. D. Atlas ambiental de Porto Alegre. Editora da Universidade, Porto Alegre, p. 297, 1999.

NIEMELÄ, J. Ecology and urban planning. Biodiversity and Conservation,v. 8, p. 11-131, 1999.

PROFOUS, G. V.; LOEB, R. E. Vegetation and plant communities of Van Cortlandt Park, Bronx, N.Y. Bulletin of Torrey Botanical Club, v. 11, p. 80-89, 1984.

PICKETT, S. T. A.; CADENASSO, M. L.; GROVE, J. M.; NILON, C. H.; PUYAT, R. V.; ZIPPERER, W. C.; CONSTANZA, R. Urban ecological eystems: linking terrestrial ecological, physical, and socioeconomic components of metropolitan areas. Landscape urban planning, v. 32, p. 127-157, 2001.

RUDNICKY, J. L.; MCDONNELL, M. J. Forty-eight years of canopy change in a hardwood hemlock forest in New York City. Bulletin of Torrey Botanical Club, v. 116, p. 52-64, 1989.

SANTOS, A. A.; RAGUSA-NETTO, J. Plant food resources exploited by Blue-and-Yellow-Macaws (Ara ararauna Linn) at an urban area in Central Brazil. Brazilian Journal of Biology. No prelo (2014).

SAVARD, J. P. L., CLERGEAU, P.; MENNECHEZ, G. Biodiversity concepts and urban ecosystems. Landscape and Urban Planning, v. 659, p.1-12, 2000.

SILVA, J. R. M. Análise e Avaliação das Erradicações na arborização urbana da cidade do Recife: percepção coletiva e apropriação social. In: CONGRESSO BRASILEIRO DE ARBORIZAÇÃO URBANA. Rio de Janeiro, Sociedade Brasileira de Arborização Urbana, 2000.

ZIPPERER, W. C.; FORESTMAN, T. W.; SISINNI, S. M.; POUYAT, R.V. Urban tree cover: an ecological perspective. Urban Ecosystems, v.1, p. 229-247, 1997. 